\title{
Stochastic effects on the establishment of mutants resistant to $\beta$-lactam antibiotics
}

\author{
Manja Saebelfeld ${ }^{1,2,}{ }^{,}$, Suman G. Das ${ }^{1,}{ }^{*}$, Arno Hagenbeek ${ }^{2}$, Joachim Krug ${ }^{1}$, \\ J. Arjan G.M. de Visser² \\ ${ }^{1}$ University of Cologne, Institute for Biological Physics, Köln, Germany \\ ${ }^{2}$ Wageningen University, Laboratory of Genetics, Wageningen, Netherlands
}

*These authors contributed equally to the work.

Corresponding authors: SG. Das sdas3@uni-koeln.de, M. Saebelfeld msaebelfeld@gmail.com 


\section{Abstract}

2 For antibiotic resistance to arise, new resistant mutants must establish in a bacterial 3 population before they can spread via natural selection. Understanding the stochastic factors

4 influencing mutant establishment is crucial for a quantitative understanding of antibiotic

5 resistance emergence. Here, we quantify the single-cell establishment probability of four

6 Escherichia coli strains expressing $\beta$-lactamase alleles with different activity against the

7 antibiotic cefotaxime, as a function of concentration. Using a simple branching model, we

8 show that concentrations well below the minimal inhibitory concentration (MIC) can

9 substantially hamper establishment, particularly for highly resistant mutants, suggesting cooperative effects due to antibiotic breakdown at high cell densities. We further show that variation among cell lineages may explain the more gradual influence of increased antibiotic concentrations on agar. Finally, we use the single-cell establishment probability to predict a resistance effects. 


\section{Introduction}

A classical question in population genetics addresses the fate of a new adaptive allele in a population. Any new allele is initially present at a low frequency in the population and thus prone to extinction by genetic drift, even if the allele is beneficial (Patwa \& Wahl, 2008). In 1927, Haldane stated that a novel beneficial allele has to overcome drift loss and establish in the population before it can be picked up by selection; the probability of which he estimated to be roughly two times its selective benefit (Haldane, 1927). Since then, Haldane's approximation has been generalised and extended by a great number of models, but none of them have been tested empirically until about a decade ago (Patwa \& Wahl, 2008). With advances in genomic techniques, the issue of fixation probability has recently been studied in experimental populations of microorganisms (e.g. Lang, Botstein, \& Desai, 2011; Frenkel, Good, \& Desai, 2014; Good, McDonald, Barrick, Lenski, \& Desai, 2017). These studies looked at the fate of beneficial alleles that are already established at a certain frequency in the population, finding that their fixation strongly depends on the competition with further beneficial alleles that appear via mutation over time. Only a small number of empirical studies has investigated the stochastic process of establishment of an initially rare beneficial allele arisen by de novo mutation in fungi (Gifford, De Visser, \& Wahl, 2013), bacteria (Farrell, Gralka, Hallatschek, \& Waclaw, 2017; Giometto, Nelson, \& Murray, 2018) and nematodes (Chelo, Nédli, Gordo, \& Teotónio, 2013). Overall, these studies found that genetic drift dominates the establishment process, and the probability for a beneficial allele to establish in a population depends on the alleles' initial frequency, its selective benefit, and the size of the population.

Whether a beneficial allele establishes successfully in a population is of particular relevance in cases of unwanted evolution, such as the evolution of antibiotic resistance. The worldwide growing concern about failing treatments of infections due to the emergence of resistant 
bacterial pathogens has led to the awareness that this problem has to be tackled from different angles, one of which is the better understanding of the emergence and evolution of antibiotic resistance (Baym, Stone, \& Kishony, 2016; Smith et al., 2016; Furusawa, Horinouchi, \& Maeda, 2018). Resistance alleles can be acquired via horizontal gene transfer or de novo mutation. In the latter case, the resistant allele is initially present in a single individual within a susceptible population. But how likely is it that this single genotype can establish in the population and what factors influence this process? Using time-lapse microscopy, Coates et al. (2018) followed the growth of single Escherichia coli cells on agar, supplemented with different antibiotics. They found that the establishment of a single cell, i.e. the growth into a visible macrocolony, was a highly stochastic process driven by fluctuations in cell death and birth, leading to the extinction of microcolonies at various time points. This stochasticity was more pronounced with increasing antibiotic concentration, resulting in higher extinction rates. Similar results were found for streptomycin resistance in Pseudomonas aeruginosa (Alexander \& MacLean, 2020). By seeding one to a few cells of a resistant strain in liquid medium with increasing antibiotic concentrations, it was found that at concentrations as low as $1 / 8$ of the minimum inhibitory concentration (MIC) of this strain, its establishment probability was only $5 \%$ and that establishment of one cell was independent of the presence of other cells in the inoculum. The authors speculated that the lack of social effects on mutant establishment may depend on the mechanism of antibiotic resistance, and could be modified when positive social interactions play a role, e.g. due to the expression of enzymes that break down the antibiotic (Alexander \& MacLean, 2020).

A common resistance mechanism potentially involving social effects involves $\beta$-lactamase enzymes. $\beta$-lactamases hydrolyse the lactam ring of $\beta$-lactam antibiotics, which target penicillin-binding proteins (PBPs) that are involved in cell wall synthesis of Gram-negative 
bacteria, thereby leading to loss of cell wall integrity and eventual cell lysis (Samaha-Kfoury \& Araj, 2003; Bush, 2010). A well-studied and clinically relevant example is TEM-1 $\beta$-lactamase, which has high activity toward first-generation penicillins, but low activity toward thirdgeneration $\beta$-lactams, including the cephalosporin cefotaxime (CTX). However, the TEM-1 allele can acquire mutations, rendering it more active to CTX (Salverda, de Visser, \& Barlow, 2010; Schenk, Szendro, Krug, \& de Visser, 2012; Van Dijk, Hwang, Krug, De Visser, \& Zwart, 2017). As $\beta$-lactamases are expressed in the periplasmic space of gram-negative pathogens like $E$. coli, this system has been recognized as potentially cooperative, since the enzymatic breakdown in the periplasm reduces the antibiotic concentration in the environment (Brown, West, Diggle, \& Griffin, 2009), thereby cross-protecting susceptible strains (Medaney, Dimitriu, Ellis, \& Raymond, 2016). Such a cooperative behaviour can be expected to be more pronounced on agar due to the local breakdown of the antibiotic, creating microenvironments that allow for co-existence of strains with different levels of antibiotic resistance (Nicoloff \& Andersson, 2016; Frost et al., 2018; Geyrhofer \& Brenner, 2020).

Here, we examined the single-cell establishment probability of antibiotic-degrading bacteria under a range of antibiotic concentrations in a structured and an unstructured environment. We used four $E$. coli strains expressing $\beta$-lactamase alleles with different activity, and hence different levels of resistance, toward CTX. Each strain was tested separately at low cell densities in both liquid and on agar medium with increasing CTX concentrations. To determine the single-cell establishment probability from the data, we developed a simple branching model, followed by a more detailed investigation of the cause of differences in stochasticity between the two environments. We show that environmental structure and cooperative behaviour play at most a minor role in the establishment of resistant cells at low initial 
densities. Based on our results, we further introduce a new measure for resistance level that takes inoculum size and stochasticity into account, in contrast to the classical MIC assay.

\section{Results and Discussion}

\section{Single-cell establishment probability}

To determine single cell establishment probabilities, we performed each one experiment in liquid medium and on agar plates. In each experiment, four E. coli strains with increasing levels of CTX resistance (referred to as Ancestor, Single mutant, Double mutant, Triple mutant; cf. Table 1) were tested separately under exposure to a range of CTX concentrations. We use the notation $p_{e}(x)$ to denote the probability that a single cell establishes a macroscopic population (i.e. growth in liquid or colony formation on agar visible with the naked eye) at CTX concentration $x$. When the antibiotic concentration is zero, a population should establish with near certainty. Likewise, when the concentration is above the strains' minimum inhibitory concentration (MIC), establishment is completely prevented. Stochastic outcomes can arise only at intermediate concentrations.

In the liquid medium experiment, we grew bacteria in multi-well plates starting from a small number of cells (1-3 on average) in each well and observed their establishment as a function of CTX concentration. Applying aliquots of the cell cultures used for inoculating the well-plates on agar, provided estimates of the viable cell numbers needed to evaluate the probabilities. Using a bootstrapping algorithm and assuming a Poisson distribution of independently acting cells in each inoculum (details in Supplement S4), we estimated the single-cell establishment probability $p_{e}(x)$ for all strains and conditions, which are shown in Figure 1. As expected, the mutants with higher resistance require higher antibiotic concentration to inhibit 
Ancestor

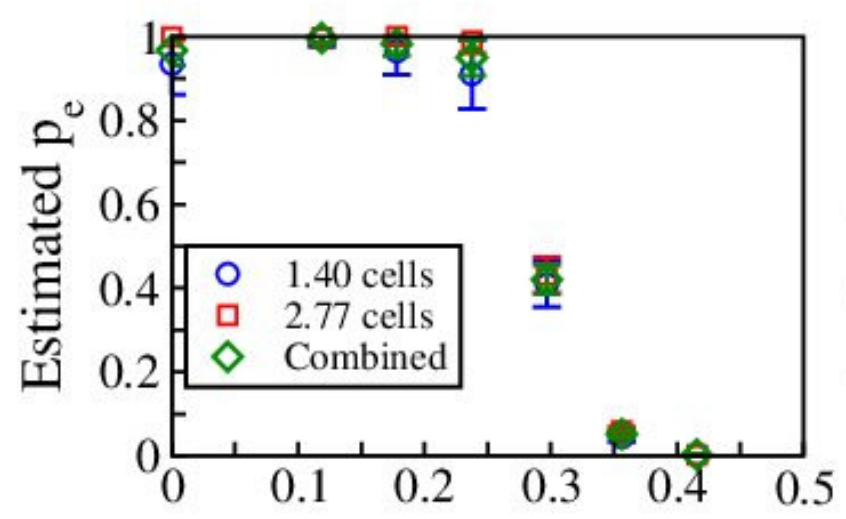

Double mutant

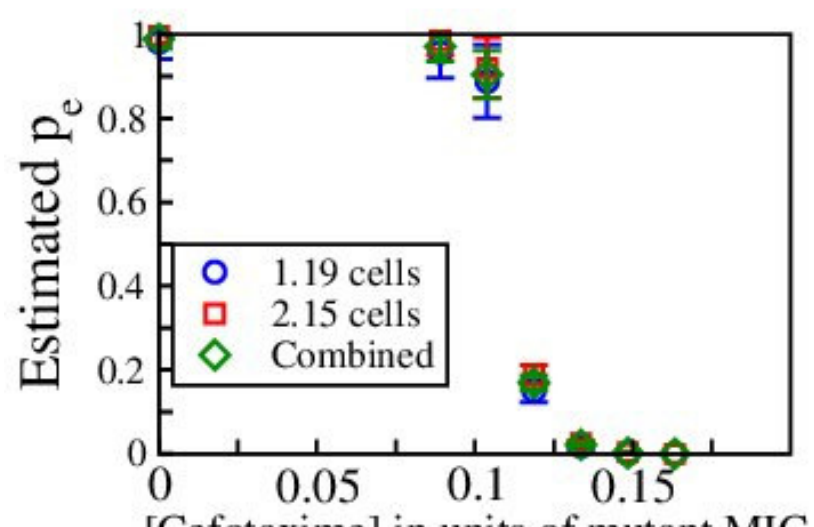

[Cefotaxime] in units of mutant MIC
Single mutant

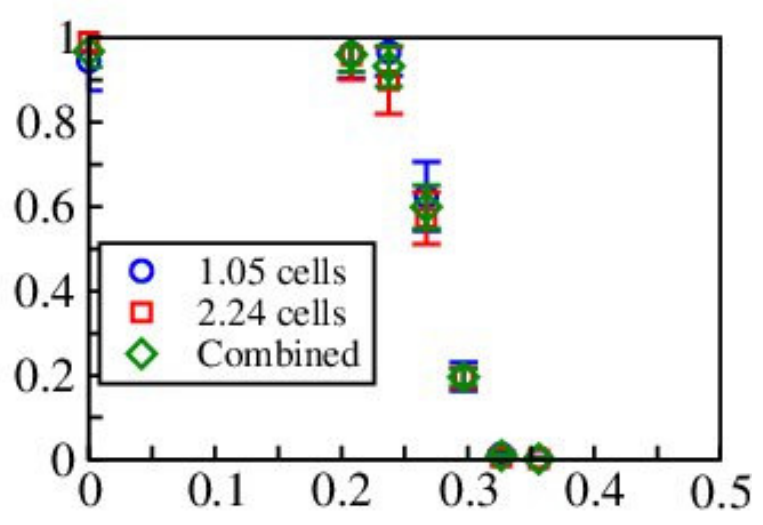

Triple mutant

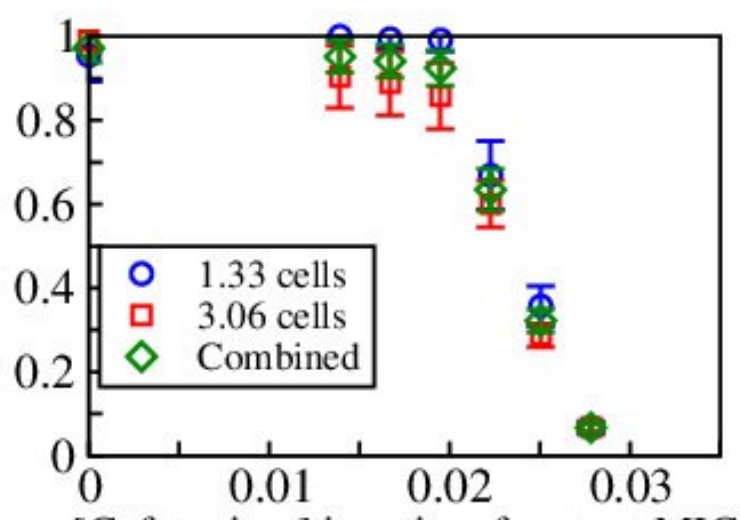

[Cefotaxime] in units of mutant MIC

Figure 1. Single-cell establishment probabilities, $p_{e}$, in liquid medium, estimated for the four tested strains as a function of antibiotic concentration. For each strain, two inocula were used, one of approximately 1 cell (blue circles) and another of 2-3 cells (red squares); average inoculum size estimated from separate CFU counts are shown. The inferred single-cell establishment probabilities are nearly identical for the two cases, implying that the establishment probability of a lineage is not influenced by the presence of other lineages at these low initial cell densities. The green diamonds are weighted averages over the two inoculum sizes (see Supplement S3 for details on the method used to estimate $p_{e}$ ).

One complication in estimating the single-cell establishment probability is that multiple cells may be present initially in some wells, and the lineages arising out of them may in principle 
In that case, the establishment probabilities of multiple lineages arising from different cells are no longer independent, biasing our estimates of $p_{e}(x)$. To test whether lineages interact from initially small inocula, we conducted the liquid experiment with two different mean inoculum sizes and estimated $p_{e}(x)$ for both cases under the assumption that there is no interaction (cf. Supplement S4). If interaction occurred, the inferred values should disagree. However, Figure 1 shows that they agree closely, indicating that interaction between lineages is insignificant at these low cell densities.

The absence of social interactions in liquid medium was also found in the study with $P$. aeruginosa and streptomycin under similar conditions regarding culture volume and inoculum size (Alexander \& MacLean, 2020). In that study, social interactions were less expected because streptomycin resistance does not involve degrading enzymes with the potential for such interactions. Possibly social interactions play a role during the establishment of new $\beta$ lactamase mutants in structured environments, where antibiotic breakdown may locally affect establishment probability.

To determine the effect of environmental structure on establishment, we plated about 200 cells of each E. coli strain on CTX-containing agar, followed by counts of colony-forming units (CFUs) after incubation. The establishment probability was estimated as the CFU count relative to that at zero concentration (Figure 2). The patterns of $p_{e}$ as a function of CTX concentration are broadly similar to those in liquid medium. Overall, these results show that the establishment process of single $E$. coli cells in our system can be described by simple branching processes, assuming a uniform cell death probability within each lineage (see Supplement S2), in both liquid and agar environments. However, when comparing the estimates for liquid and agar medium more closely, one notices that the decline of $p_{e}$ with CTX concentration is less steep on agar than in liquid, even though the average establishment 
Ancestor

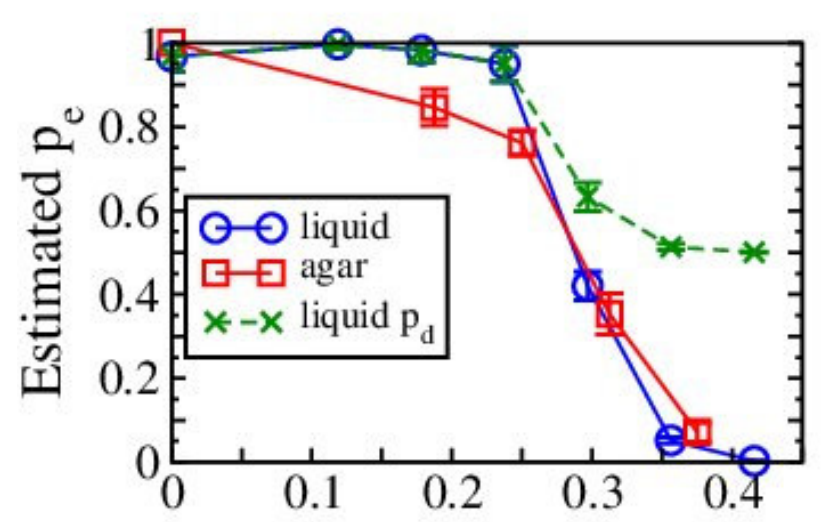

Double mutant

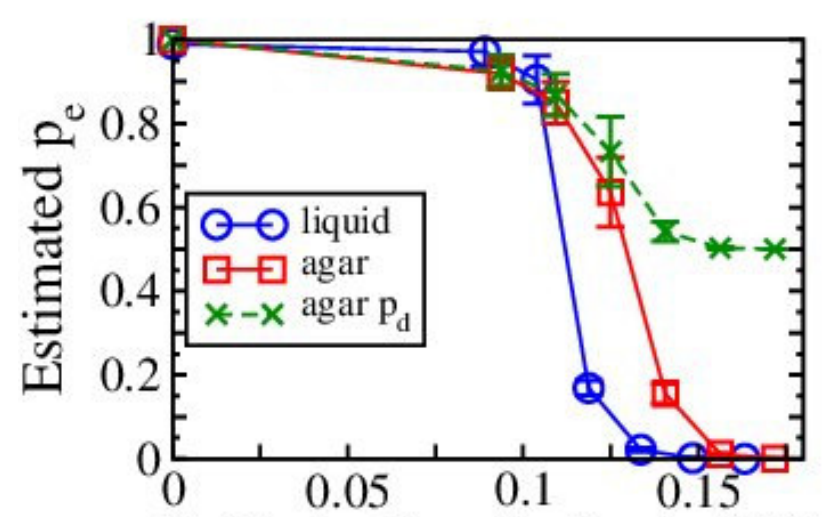

[Cefotaxime] in units of mutant MIC
Single mutant

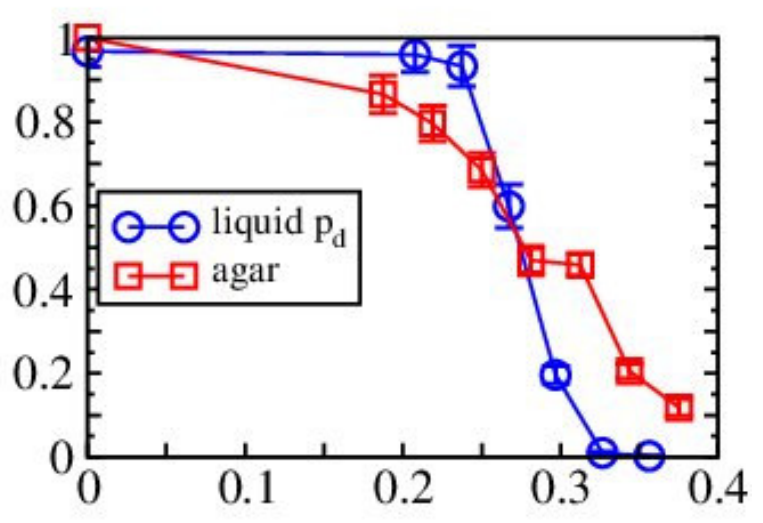

Triple mutant

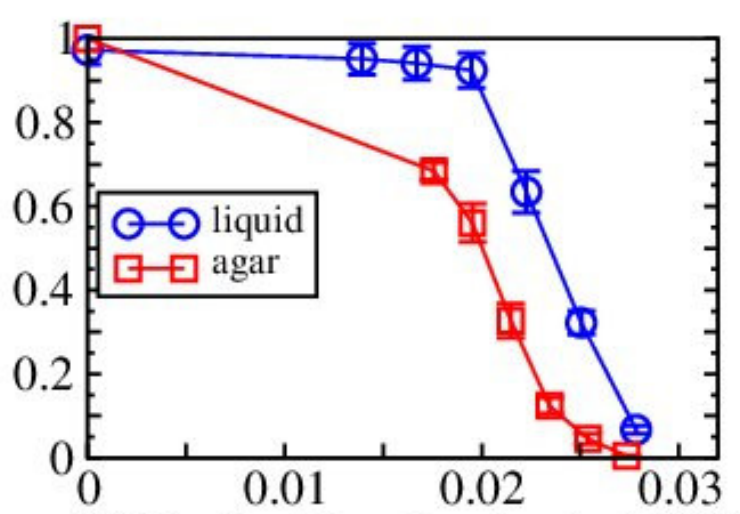

[Cefotaxime] in units of mutant MIC
153

Figure 2. Estimated single-cell establishment probabilities for the four strains as a function of CTX concentration in liquid and on agar. For liquid, the values are obtained as weighted averages over the two inoculum sizes (as shown in Figure 1). For agar, the inoculum size is effectively 1 , since each colony usually grows from a single cell. Green dots (with dashed lines) show the per-cell division probability, $p_{d}$, (estimated using the branching model without lineage variability), for two particular strains, one each for liquid and agar. The shape of the $p_{d}$ curve is similar for the other cases (not shown).

\section{Sources of stochasticity and the branching model}

When the establishment probability is different from 0 or 1 , it means that the colony growth process arising from genetically identical cells in the same environment can have different 
outcomes. There can be two different sources of stochasticity in the system. One source we call inherent noise, which arises from random thermal fluctuations in chemical reactions. Two nascent cells that are identical in structure and composition may still have different establishment fates due to inherent noise, potentially leading to different outcomes like cell division for one and cell lysis for another. The other source we call lineage variability, which we define as the systematic variation between founder cells of colonies, that can arise from phenotypic differences already present between cells in the inoculum or can be caused by small variations across the micro-environments in which the colonies grow. We aim to differentiate between the two sources of stochastic effects in our data and understand the relative contribution of each in the two tested environments with a different structure.

To do so, we model population growth as a branching process, where each cell has a cell division probability, $p_{d}$, and consequently a cell death probability, $1-p_{d}$. Inherent noise is present whenever $p_{d}$ is different from 0 and 1 and we assume all cells in a lineage to have the same $p_{d}$. In a branching process, the simplest definition of establishment is that the population continues to be of non-zero size indefinitely. For our branching model,

$$
p_{e}=2-\frac{1}{p_{d}}
$$

when $p_{d} \geq \frac{1}{2}$, and $p_{e}=0$ when $p_{d} \leq \frac{1}{2}$ (see Supplement S2 for details). Considering $p_{d}$ to be identical for all cell lineages rules out lineage variation, and cell fate is purely a result of inherent noise. To see if this is sufficient to explain the data, we estimate $p_{d}$ from the observed values of $p_{e}$, which are shown in Figure 2 . The way $p_{d}$ approaches $\frac{1}{2}$ asymptotically with increasing concentrations is counter to the expectation that $p_{d} \rightarrow 0$ at very high concentration. This indicates either that our definition of establishment is not biologically realistic, or that inherent noise is inadequate for explaining the intermediate values of $p_{e}$. We can modify the definition of establishment to make it more realistic by requiring that the process reaches a 
certain threshold cell number in the time window of the experiment (Schenk et al., 2012). The results (shown in Supplement S2) indicate that this is not sufficient to explain the data. We, therefore, need to include lineage variability into our model.

\section{Model for $p_{e}(x)$}

To arrive at a model that incorporates both sources of stochasticity, inherent noise and lineage variability, we start by modelling $p_{d}$ as a Hill function of the antibiotic concentration, $x$, and the antibiotic concentration where $p_{d}$ is $1 / 2, x_{0}$ :

$$
p_{d}\left(x, x_{0}\right)=\frac{1}{1+\left(\frac{x}{x_{0}}\right)^{n}}
$$

A mechanistic basis for this functional form is as follows: due to inactivation of penicillinbinding proteins (PBPs, which are the main target of CTX; Rossolini, Arena, \& Giani, 2017), there is a shortage of PBP molecules, leading to the formation of cell wall pores, some of which can eventually lead to bulging of the cell wall and lysis (Yao, Kahne, \& Kishony, 2012). Let the number of pores be Poisson distributed with mean $\lambda$, and let the probability that a pore does not bulge be $q$. Then $p_{d}$ is the probability that there are no bulging pores, which can be shown to be equal to $e^{-\lambda(1-q)}$. The dependence of $\lambda$ on the concentration is not known, but the choice of a simple empirical form for $\lambda$ produces the function in (2) (see Supplement S5 for details). We emphasize that the choice of the Hill function is not unique; any inverted sigmoidal function would suffice for the purpose. Note that in (2), $x_{0}$ is the concentration at which $p_{d}$ drops to $\frac{1}{2}$, and consequently $p_{e}$ drops to 0 ; in other words, $x_{0}$ is the single-cell MIC (Artemova, Gerardin, Dudley, Vega, \& Gore, 2015). The value of $x_{0}$ is determined in part by the level of $\beta$-lactamase activity, as a higher antibiotic degradation rate would produce a higher $x_{0}$. The quantity $\frac{x}{x_{0}}$ can be interpreted as the effective (dimensionless) intracellular antibiotic concentration. The Hill exponent $n$ serves as a measure of the inherent noise. In the limit $n \rightarrow$ 
$\infty, p_{d}(x)$ behaves like a step function that drops sharply from 1 to 0 at $x=x_{0}$, implying no stochasticity.

Lineage variability, on the other hand, is introduced via among-lineage variation in the parameter $x_{0}$. Specifically, we model $x_{0}$ as a random variable drawn independently for each lineage from the gamma distribution:

$$
P\left(x_{0}\right)=\frac{1}{\Gamma(k) a^{k-1}} x_{0}^{k-1} e^{\frac{-x_{0}}{a}}
$$

The mean of $x_{0}$ is $k a$, and the variance is $k a^{2}$. The parameter $a$ provides a scaling factor of $x_{0}$. The coefficient of variation of $x_{0}$ is a measure of lineage variability, which in this case is

$k^{-\frac{1}{2}} ; k$ is therefore inversely related to the degree of lineage variability. The single-cell establishment probability inferred from a large number of observations is then simply the establishment probability (1) averaged over the distribution of $x_{0}$. The final model for establishment probability is then:

$$
p_{e}(x)=\int_{x}^{\infty} d x_{0} P\left(x_{0}\right)\left(1-\left(\frac{x}{x_{0}}\right)^{n}\right)
$$

The parameter $n$ for intrinsic noise is the same for all mutants, but may, in general, differ between liquid and agar environments. The parameters for the distribution of $x_{0}$ can be different for the tested strains and can depend on the environment as well. We performed collective fits using the same value of $n$ for both media, shown in Figure 3. It was estimated that $n=7.18 \pm 0.60$ and the coefficient of variation on agar was higher than in liquid for most strains, consistent with the less steep curve for agar. In Figure 3, the $x$-values for each 
strains largely collapse, indicating small (but non-zero) lineage variability in $x_{0}$. The coefficients of variation of $x_{0}$, which contain the effect of lineage variability, are larger for the agar than for the liquid environment in most cases, as expected, but the difference is small.

We also performed model fits with separate estimates of $n$ for liquid and agar. In this case, details), making it hard to distinguish between the contribution of both sources of

is more parsimonious, and higher lineage variability on agar compared to liquid may also be

A

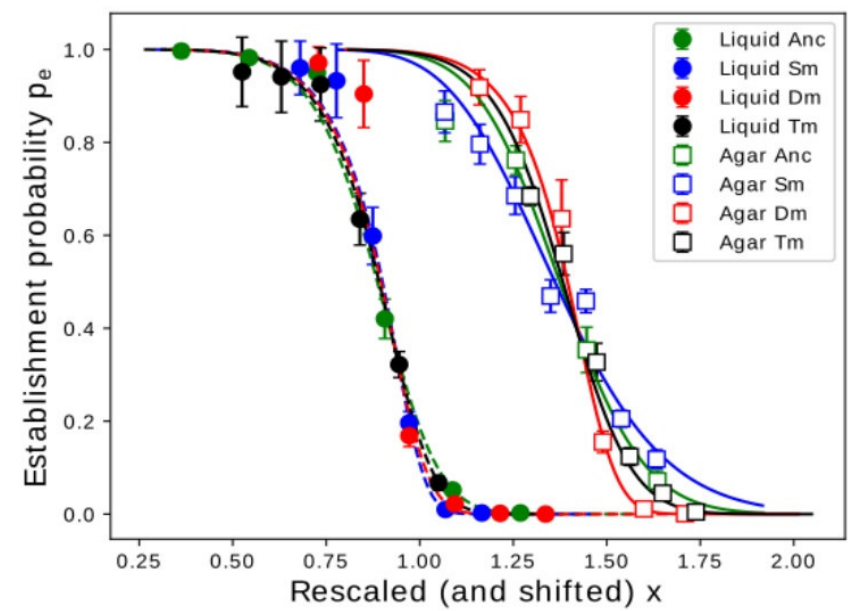

\section{B}

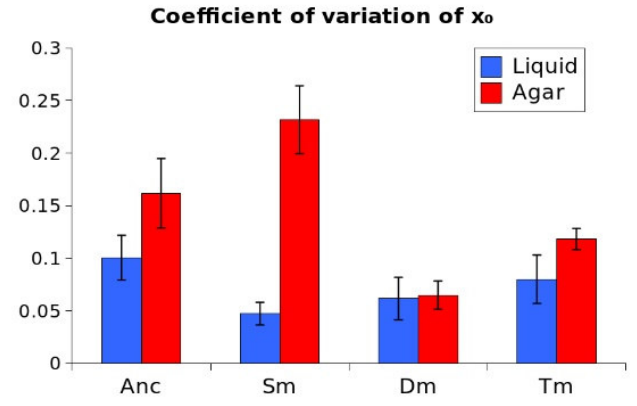

Figure 3. Effect from intrinsic noise and lineage variability in $x_{0}$ on single-cell establishment probability in liquid compared to agar. A joint fit of equation (3) with the same value for $n$ for both environments was used. A: $p_{e}(x)$ obtained from curve-fitting. The $x$-values for each strain (Anc: TEM-1 Ancestor, Sm: Single mutant, Dm: Double mutant, Tm: Triple mutant) have been scaled by their respective mean $x_{0}$, and the values for agar have been shifted along the $x$-axis to the right by 0.5 to improve visibility only. B: The coefficient of variation of $x_{0}$ (mean \pm S.D.), indicating higher lineage variability on agar than in liquid for most strains. 
One of the main findings in the $P$. aeruginosa study mentioned above (Alexander \& MacLean, 2020), was that the used mutant strain, when seeded alone at a few cells in a similar setup as ours, showed a $<5 \%$ establishment probability at a streptomycin concentration of $1 / 8$ of the strain's MIC. At the same MIC fraction, the establishment probabilities for our strains are about 100, 100, 8 and 0\% for the Ancestor, Single mutant, Double mutant and Triple mutant, respectively. These results show that there is no general relationship between the MIC and the single-cell establishment probability, neither across systems nor among genotypes of the same species within the same system. The cause for this is likely rooted in how the MIC is measured. In a standard MIC assay, $10^{5}$ cells are inoculated into $200 \mu \mathrm{l}$ medium in a series of wells with 2-fold differences in antibiotic concentration. Thus, the "real" MIC value lies between the smallest concentration where no growth is observed (defined as MIC) and half this concentration. The concentration range of where the real MIC lies, is then smaller or larger depending on the MIC of the respective strain. Another issue with the MIC is that even the growth of an inoculum as large as $10^{5}$ cells is a stochastic process under sufficiently high antibiotic concentrations (Coates et al., 2018), which generally results in a reduced reproducibility of MIC assays.

A conceptually cleaner approach to determine the MIC would be to recognise this stochasticity. Here, we introduce a new measure, $M I C_{q}(N)$, defined as the concentration at which an inoculum of size $N$ has a probability $q$ of not becoming established. In the absence of interactions between lineages, $M I C_{q}(N)$ can be predicted from $p_{e}(x)$ (see Supplement $\mathrm{S} 7$

277 for details). Setting $q=\frac{1}{2}$ and $N=10^{5}$, we obtain $M I C_{q}(N)$ values of $0.038,0.23,1.6$, and $2782.9 \mu \mathrm{g} / \mathrm{ml} \mathrm{CTX}$ for the Ancestor, Single mutant, Double mutant and Triple mutant, respectively.

279 The values are smaller than the previously determined MICs (Table 1), and the differences 
become increasingly pronounced with the level of resistance of the mutants. This suggests

281

282

283

that positive interactions among cell lineages enhance their collective establishment due to the enzymatic breakdown of the antibiotic in the medium, which is particularly pronounced at high cell densities and for cells expressing enzymes with high activity against CTX.

The effect of inoculum size on the MIC through social interactions has been recognised before (Brook, 1989; Tan et al., 2012). Causes for such social effects have been suggested to include binding of the antibiotic to cellular components (Queenan, Foleno, Gownley, Wira, \& Bush, 2004) or the expression of proteins that inactivate the antibiotic (Lenhard \& Bulman, 2019). Our new measure includes a purely statistical inoculum effect, which leads to a logarithmic increase of $M I C_{q}(N)$ for large $N$ (see Supplement S7 for details). Our results on the discrepancy between the MIC and the single-cell establishment probability are in line with the findings of other studies that criticised the MIC assay as not suitable for dosage determination for the treatment of antibiotic-resistant infections (Gould \& MacKenzie, 1997; Artemova et al., 2015).

\section{Conclusion}

Our study contributes to the understanding of the establishment of adaptive mutants, a topic which has been under much theoretical scrutiny but is rarely investigated empirically. We show that establishment of populations starting from a single cell in the presence of an antibiotic can be well described by a simple branching model both in liquid and agar environments, where the cell division probability is dependent on the antibiotic concentration. We find that the establishment of single mutant cells is negatively affected by CTX concentrations well below each tested strains' MIC, consistent with previous findings(Alexander \& MacLean, 2020). 
One aim of our study was to test if cooperative behaviour (i.e. collective antibiotic breakdown) among cells of the same strain would affect establishment probability and whether such an effect differed between unstructured and structured environments. Our data show a reasonable agreement between establishment in liquid and agar media, indicating that environmental structure plays only a modest role, and that cooperative behaviour has a negligible effect on the establishment probability of single resistant mutant cells. However, the $p_{e}(x)$ curves for agar are slightly shallower than for the liquid environment, indicating higher stochasticity. By evaluating the effect of two potential sources of stochasticity, random intrinsic noise and systematic (e.g. heritable) variability among cell lineages, we found that the simplest explanation for this observation is an enhanced lineage variability on agar, possibly arising from small differences in local agar conditions.

Lastly, we show that social interaction effects on the establishment of antibiotic-resistant mutants can be strong at high cell densities, making conventional MIC values highly sensitive to the assay conditions. We suggest a more robust measure of resistance which includes both inoculum size and probabilistic effects, $M I C_{q}(N)$, and can be compared easily with $p_{e}$ to test for the presence of interactions.

\section{Material and Methods}

\section{$\underline{\text { Strains and culture conditions }}$}

Four strains were used for the experiments, derived from the Escherichia coli strain MG1655 DA28100. This strain had previously been modified with a YFP (yellow fluorescent protein) marker cassette, containing a resistance gene for chloramphenicol (Gullberg, Albrecht, Karlsson, Sandegren, \& Andersson, 2014). For the current study, the chloramphenicol resistance was removed, and each one of four TEM variants was inserted into the galK locus: 


\begin{tabular}{ccccc}
\hline $\begin{array}{c}\text { Wildtype strain } \\
\text { (without TEM } \\
\text { allele) }\end{array}$ & $\begin{array}{c}\text { TEM amino acid } \\
\text { substitution }\end{array}$ & MIC for Cefotaxime $(\boldsymbol{\mu g} / \mathbf{m l})$ & $\begin{array}{c}\text { Strain name } \\
\text { (as used here) }\end{array}$ \\
\cline { 2 - 5 } & MIC* & $\boldsymbol{M I C}_{\boldsymbol{q}}(\boldsymbol{N})^{* *}$ & \\
MG1655 & G238S & 0.08 & 0.038 & Ancestor \\
\cline { 2 - 5 } DA28100 & 0.64 & 0.23 & Single mutant \\
\cline { 2 - 5 } galK::YFPDCAT- & E104K/G238S & 10.24 & 1.6 & Double mutant \\
\cline { 2 - 5 } & E104K/M182T/G238S & 81.92 & 2.9 & Triple mutant \\
\hline
\end{tabular}

the ancestral allele TEM-1 (referred to as "Ancestor" strain), and three mutant alleles with either 1, 2 or 3 point mutations in the TEM allele (referred to as "Single mutant", "Double mutant" and "Triple mutant", respectively). While the TEM-1 ancestral allele confers only low activity towards the $\beta$-lactam antibiotic cefotaxime (CTX), the three mutants show increasing CTX resistance with each additional substitution (Table 1). All TEM loci are under the control of the lacl repressor and are expressed by adding $50 \mu \mathrm{M}$ Isopropyl $\beta-\mathrm{D}-1$ thiogalactopyranoside (IPTG) to the growth medium. The minimum inhibitory concentration (MIC) of each strain for CTX had previously been determined in duplicates (Table 1), using 2fold increases in CTX concentration in microtiter plates filled with $200 \mu \mathrm{l}$ M9 minimal medium (containing $0.4 \%$ glucose, $0.2 \%$ casaminoacids, $2 \mu \mathrm{g} / \mathrm{ml}$ uracil and $1 \mu \mathrm{g} / \mathrm{ml}$ thiamine) and 50 $\mu \mathrm{M}$ IPTG, inoculated with $10^{5}$ cells and incubated for $24 \mathrm{~h}$ at $37^{\circ} \mathrm{C}$.

For culturing, all strains were first recovered from glycerol stocks by streaking them out on each one LB (Luria-Bertani) agar plate and incubated overnight at $37^{\circ} \mathrm{C}$. From those plates, each one colony was picked into $1 \mathrm{ml} \mathrm{M9}$ medium (as above) and incubated overnight at $37^{\circ} \mathrm{C}$, $250 \mathrm{rpm}$. The cultures were then serially diluted with phosphate-buffered saline (PBS) to the density needed for the particular experiment (see below), assuming an initial density of $2.5 \times 10^{9}$ cells $/ \mathrm{ml}$.

\footnotetext{
*As determined prior to the study by standard MIC assays (see Material and Methods).
} 
${ }^{* *}$ As predicted from $p_{e}(x)$ in the current study (see the last section of Results and Discussion, and Supplement S7).

\section{Liquid experiment}

Each of the four strains was tested separately in the absence of CTX and six CTX concentrations

(Supplement S1: Table S1), where the particular tested concentrations depended on the strains' resistance level. The range of concentrations was chosen to display establishment probabilities between 0 and 1 , based on pilot experiments to find the right conditions (data not shown).

Per strain and CTX concentration, on average either about 1 or 2 cells were seeded into 285 wells across three 96-well microtiter plates. For this, $190 \mu \mathrm{l}$ M9 medium with the respective cefotaxime concentration and $50 \mu \mathrm{M}$ IPTG were pipetted into all wells. Overnight cultures of the strains were diluted to 200 and 100 cells/ml with PBS. $10 \mu$ of the dilutions were pipetted into the wells. One well per plate served a medium control, mock-inoculated with $10 \mu \mathrm{PBS}$. The plates were incubated about $40 \mathrm{~h}$ at $37^{\circ} \mathrm{C}$ (static). After incubation, $\mathrm{OD}$ at $600 \mathrm{~nm}$ of all wells was measured in a plate reader (Victor $3^{\text {TM }}$, PerkinElmer) without the plate lid. All medium controls across the experiment showed no sign of growth; the OD ranged from 0.033 to 0.038 . Thus, a threshold of $>0.05$ was applied to determine whether a well successfully established (i.e. showed growth).

To test for correct culture dilutions and Poisson distribution of seeded cell numbers, the same culture dilutions used for seeding the well plates were dropped on LB agar plates $(120 \times 120$ $\mathrm{mm})$. Per dilution and strain, a total of $3 \times 3210 \mu \mathrm{l}$ drops were applied to the plates, let dry for about 10 to 20 minutes, incubated at $37^{\circ} \mathrm{C}$ until leaving, and then moved to $30^{\circ} \mathrm{C}$ overnight to prevent overgrowth, followed by counting the number of colonies per droplet. 
373

374

375

376

377

378

379

380

381

382

383

384

385

386

387

388

389

390

391

392

393

394

395

\section{Agar experiment}

As in the liquid experiment, all strains were tested separately and the CTX concentration range depended on the strain. Five to eight concentrations were tested per strain (Supplement S1: Table S1). The overnight cultures were diluted to 4000 cells $/ \mathrm{ml} .50 \mu \mathrm{l}$ ( 200 cells) of the dilutions were spread onto $92 \mathrm{~mm}$ agar plates containing M9 medium (as above) with $1.5 \%$ agar, $50 \mu \mathrm{M}$ IPTG and the respective cefotaxime concentration, using a bacterial spreader. Per strain and cefotaxime treatment, eight replicate plates were used. The plates were incubated at $37^{\circ} \mathrm{C}$ until colonies were big enough to count them unambiguously (20 to 48 hours). Each colony is regarded as a successfully established single cell.

\section{Acknowledgements}

We would like to thank Helen Alexander for fruitful discussions of her work and advice on the liquid experiment setup; and Tobias Bollenbach for advice on the $p_{e}$ model.

\section{Author contribution}

All authors conceived the study. MS and AH conducted the experiments. SGD developed the models with help from JK. MS and SGD wrote the manuscript with help from JK and JAGMdV. All authors read the manuscript and approved the final version.

\section{Competing Interests Statement}

All authors declare that they have no significant competing financial, professional, or personal interests that might have influenced the performance or presentation of the work described in this manuscript. 


\section{References}

Alexander, H. K., \& MacLean, R. C. (2020). Stochastic bacterial population dynamics restrict the establishment of antibiotic resistance from single cells. Proceedings of the National Academy of Sciences of the United States of America, 117(32), 19455-19464. https://doi.org/10.1073/pnas.1919672117

Artemova, T., Gerardin, Y., Dudley, C., Vega, N. M., \& Gore, J. (2015). Isolated cell behavior drives the evolution of antibiotic resistance. Molecular Systems Biology, 11(7), 822. https://doi.org/10.15252/msb.20145888

Baym, M., Stone, L. K., \& Kishony, R. (2016). Multidrug evolutionary strategies to reverse antibiotic resistance. Science, 351(6268). https://doi.org/10.1126/science.aad3292

Brook, I. (1989). Inoculum Effect. Reviews of Infectious Diseases, 11(3), 361-368.

$$
\text { https://doi.org/10.1093/clinids/11.3.361 }
$$

Brown, S. P., West, S. A., Diggle, S. P., \& Griffin, A. S. (2009). Social evolution in microorganisms and a Trojan horse approach to medical intervention strategies. Philosophical Transactions of the Royal Society B: Biological Sciences, 364(1533), 3157-3168. https://doi.org/10.1098/rstb.2009.0055

Bush, K. (2010). Bench-to-bedside review: the role of $\beta$-lactamases in antibiotic-resistant Gram-negative infections. Critical Care, 14(3), 224.

Chelo, I. M., Nédli, J., Gordo, I., \& Teotónio, H. (2013). An experimental test on the probability of extinction of new genetic variants. Nature Communications, 4, 1-8. https://doi.org/10.1038/ncomms3417

Coates, J., Park, B. R., Le, D., Şimşek, E., Chaudhry, W., \& Kim, M. (2018). Antibiotic-induced population fluctuations and stochastic clearance of bacteria. ELife, 7, 1-26. https://doi.org/10.7554/eLife.32976

Farrell, F. D., Gralka, M., Hallatschek, O., \& Waclaw, B. (2017). Mechanical interactions in 

bacterial colonies and the surfing probability of beneficial mutations. Journal of the Royal Society Interface, 14(131). https://doi.org/10.1098/rsif.2017.0073

Frenkel, E. M., Good, B. H., \& Desai, M. M. (2014). The fates of mutant lineages and the distribution of fitness effects of beneficial mutations in laboratory budding yeast populations. Genetics, 196(4), 1217-1226. https://doi.org/10.1534/genetics.113.160069

Frost, I., Smith, W. P. J., Mitri, S., San Millan, A., Davit, Y., Osborne, J. M., ... Foster, K. R. (2018). Cooperation, competition and antibiotic resistance in bacterial colonies. ISME Journal, 12(6), 1582-1593. https://doi.org/10.1038/s41396-018-0090-4

Furusawa, C., Horinouchi, T., \& Maeda, T. (2018). Toward prediction and control of antibiotic-resistance evolution. Current Opinion in Biotechnology, 54, 45-49. https://doi.org/10.1016/j.copbio.2018.01.026

Geyrhofer, L., \& Brenner, N. (2020). Coexistence and cooperation in structured habitats. BMC Ecology, 20(1), 1-15. https://doi.org/10.1186/s12898-020-00281-y

Gifford, D. R., De Visser, J. A. G. M., \& Wahl, L. M. (2013). Model and test in a fungus of the probability that beneficial mutations survive drift. Biology Letters, 9(1). https://doi.org/10.1098/rsbl.2012.0310

Giometto, A., Nelson, D. R., \& Murray, A. W. (2018). Physical interactions reduce the power of natural selection in growing yeast colonies. Proceedings of the National Academy of Sciences of the United States of America, 115(45), 11448-11453. https://doi.org/10.1073/pnas.1809587115

Good, B. H., McDonald, M. J., Barrick, J. E., Lenski, R. E., \& Desai, M. M. (2017). The dynamics of molecular evolution over 60,000 generations. Nature, 551(7678), 45-50. https://doi.org/10.1038/nature24287 
Gould, I. M., \& MacKenzie, F. M. (1997). The response of Enterobacteriaceae to beta-lactam antibiotics-'round forms, filaments and the root of all evil'. Journal of Antimicrobial Chemotherapy, 40(4), 495-499.

Gullberg, E., Albrecht, L. M., Karlsson, C., Sandegren, L., \& Andersson, D. I. (2014). Selection of a multidrug resistance plasmid by sublethal levels of antibiotics and heavy metals. MBio, 5(5), 19-23. https://doi.org/10.1128/mBio.01918-14.Editor

Haldane, J. B. S. (1927). A mathematical theory of natural and artificial selection, Part V: selection and mutation. Mathematical Proceedings of the Cambridge Philosophical Society, 23(7), 838-844. https://doi.org/10.1017/S0305004100015644

Lang, G. I., Botstein, D., \& Desai, M. M. (2011). Genetic variation and the fate of beneficial mutations in asexual populations. Genetics, 188(3), 647-661. https://doi.org/10.1534/genetics.111.128942

Lenhard, J. R., \& Bulman, Z. P. (2019). Inoculum effect of $\beta$-lactam antibiotics. The Journal of Antimicrobial Chemotherapy, 74(10), 2825-2843. https://doi.org/10.1093/jac/dkz226

Medaney, F., Dimitriu, T., Ellis, R. J., \& Raymond, B. (2016). Live to cheat another day: bacterial dormancy facilitates the social exploitation of $\beta$-lactamases. ISME Journal, 10(3), 778-787. https://doi.org/10.1038/ismej.2015.154

Nicoloff, H., \& Andersson, D. I. (2016). Indirect resistance to several classes of antibiotics in cocultures with resistant bacteria expressing antibiotic-modifying or -degrading enzymes. Journal of Antimicrobial Chemotherapy, 71(1), 100-110. https://doi.org/10.1093/jac/dkv312

Patwa, Z., \& Wahl, L. M. (2008). The fixation probability of beneficial mutations. Journal of the Royal Society Interface, 5(28), 1279-1289. https://doi.org/10.1098/rsif.2008.0248 
$\beta$-lactamase activity in ampC- and extended-spectrum $\beta$-lactamase (ESBL)-producing Escherichia coli and Klebsiella pneumoniae clinical isolates tested by using NCCLS ESBL Methodology. Journal of Clinical Microbiology, 42(1), 269-275. https://doi.org/10.1128/JCM.42.1.269

Rossolini, G. M., Arena, F., \& Giani, T. (2017). Mechanisms of antibacterial resistance. Infectious Diseases (Fourth Edi). Elsevier Ltd. https://doi.org/10.1016/b978-0-7020$6285-8.00138-6$

Salverda, M. L. M., de Visser, J. A. G. M., \& Barlow, M. (2010). Natural evolution of TEM-1 $\beta$ lactamase: experimental reconstruction and clinical relevance. FEMS Microbiology Reviews, 34(6), 1015-1036. https://doi.org/10.1111/j.1574-6976.2010.00222.x

Samaha-Kfoury, J. N., \& Araj, G. F. (2003). Recent developments in $\beta$ lactamases and extended spectrum $\beta$ lactamases. Bmj, 327(22 November 2003), 1209-1213. https://doi.org/10.1136/bmj.327.7425.1209

Schenk, M. F., Szendro, I. G., Krug, J., \& de Visser, J. A. G. M. (2012). Quantifying the adaptive potential of an antibiotic resistance enzyme. PLoS Genetics, 8(6), e1002783. https://doi.org/10.1371/journal.pgen.1002783

Smith, E., Lichten, C. A., Taylor, J., MacLure, C., Lepetit, L., Harte, E., ... Littmann, J. (2016). Evaluation of the EC action plan against the rising threats from antimicrobial resistance.

Tan, C., Phillip Smith, R., Srimani, J. K., Riccione, K. A., Prasada, S., Kuehn, M., \& You, L. (2012). The inoculum effect and band-pass bacterial response to periodic antibiotic treatment. Molecular Systems Biology, 8(617), 1-11. https://doi.org/10.1038/msb.2012.49

Van Dijk, T., Hwang, S., Krug, J., De Visser, J. A. G. M., \& Zwart, M. P. (2017). Mutation supply and the repeatability of selection for antibiotic resistance. Physical Biology, 14(5). 
